\title{
DES VOIES POUR LA RECHERCHE SUR LA RADIO
}

\section{Frédéric Antoine ${ }^{1}$}

Dans l'introduction de l'ouvrage qu'il consacrait à la radio en France en 1997, Jean-Jacques Cheval relevait les titres de publications significatives consacrées à ce média au cours des dernières années : $L a$ radio, introduction à un média méconnu ${ }^{2}$; Le medium invisible, la radio publique, commerciale et communautaire ${ }^{3}$; Radio : le média oublié ${ }^{4}$. Suite à cette énumération, Jean-Jacques Cheval écrivait, avec regret ${ }^{5}$ :

Tour à tour et d'un pays à l'autre, ces titres semblent s'accorder pour souligner combien la radio est passée au second plan des préoccupations éditoriales et scientifiques et comment ce média, si présent encore, paraît l'objet de peu d'attention ${ }^{6}$.

1 Professeur à l'UCL, aux FNDP, aux FUCAM et à l'IAD, École de Journalisme de Louvain, Observatoire du Récit Médiatique UCL, coordinateur du colloque « Radio et recherche : quelles voix/voies pour le futur de la radio » et du présent dossier de Recherches en Communication

2 Faus-Belau Angel, Espagne, 1981.

3 Lewis Peter et Booth Jerry, Grande-Bretagne, 1989.

4 Pease Edward C. et Dennis Everette E., États-Unis, 1995.

5 Introduisant son article de la revue Hermès sur «La radio, un média délaissé » ( ${ }^{\circ} 38$, pp. 63-69, 2004), il est à noter que Jean-François Tetu fait lui aussi référence aux travaux cités par Jean-Jacques Cheval.

6 Cheval Jean-Jacques, Les radios en France, Rennes, Apogée, 1997, p. 9.

Recherches en communication, $\mathrm{n}^{\circ} 26$ (2006). 
Introduisant trois années plus tard un ouvrage anglo-saxon sur l'étude de la radio, Stephen Barnard développait à peu près le même constat, écrivant ${ }^{1}$ :

Radio has been a neglected field within the Media Studies over the years $(. .$.$) .$

Toutefois, cet auteur complétait la phrase par d'autres mots :

but there is a growing litterature. Academic approaches to radio have drawn on many disciplines : cultural studies, linguistic analysis, political and economic history, sociology, psychology, business studies and marketing, even literary criticism...

Un renouveau pour l'étude de la radio semble en effet avoir vu le jour dès le tournant des années 2000 aux USA ainsi que dans le monde anglo-saxon. Et, ces derniers temps, un nouvel intérêt scientifique et académique pour la radio est devenu tangible en Europe. L'augmentation du nombre de jeunes chercheurs intéressés par l'étude des médias, et leur tendance de plus en plus marquée à se spécialiser dans des champs jusqu'ici moins encombrés des sciences de l'information et de la communication ne sont assurément pas étrangères à ce « retour » de la radio. Mais ces éléments n'expliquent pas, à eux seuls, qu'un média aussi largement délaissé par la recherche et l'enseignement pendant des décennies suscite à nouveau désormais la curiosité des analystes.

La pérennité du média radiophonique représente sans doute une des raisons de fond de l'attention cyclique qu'on lui porte dans les milieux académiques : alors que de nouveaux moyens de communication de masse voient sans cesse le jour et que la recherche se focalise naturellement sur les plus accessibles et les plus visibles d'entre eux, la place occupée par l'usage domestique de la radio reste importante dans les régions favorisées du globe et, bien plus encore, dans les zones toujours en développement.

1 Barnard Stephen, Studying Radio, London New York, Arnold-Oxford University Press, 2000, p. 3. 
Les renouvellements répétés vécus par le média radio au cours de ces dernières années ne sont assurément eux aussi pas étrangers au regain d'intérêt qu'il suscite. La radio du début de ce vingt-et-unième siècle, polymorphe et polyfonctionnelle, n'entretient plus que de très lointains rapports avec le média généraliste, guindé et pérorant que l'on connut jadis.

Enfin, la radio bénéficie aujourd'hui de l'immense avantage de ne plus devoir considérer que son avenir est derrière elle. Les évolutions technologiques en cours sont sur le point d'engager les modes de transmission sociale des messages sonores sur de nouvelles voies, impensables encore il y a peu. Et ces nouveaux modes de communication radiophoniques garantissent d'ores et déjà à la radio d'autres lendemains qui chantent.

« La » radio est morte. Vive « les » radios. Jadis il y avait « une » radio, générant autour d'elle un champ d'analyses relativement circonscrit, et en grande partie abouti. Désormais, il faut parler « des » radios, et autour d'elles d'autant de nouveaux champs d'investigation et de problématisation.

Ces conclusions, tirées au lendemain de deux jours de colloque international sur le futur de l'étude de la radio tenus à l'Université Catholique de Louvain (UCL) en novembre 2006, résument la révolution qui s'est emparée ces dernières années de l'étude de ce média et les promesses d'avenir que celle-ci laisse deviner. Car il n'y aura plus demain un ou deux modèles radiophoniques, mais des dizaines de modes d'écoute de sons, de musiques et de paroles. Même si l'un d'entre eux, lié à la magie du direct, subsistera sans doute davantage que les autres.

Colloque de clôture des travaux du Consortium Européen de recherche IREN (International Radio Research Network) soutenu par l'Union Européenne, la réunion qui s'est déroulée à l'UCL avait réuni plus de 120 scientifiques et académiques spécialisés autour de la question suivante : «Quelles voix/voies pour le futur de la radio ?».

Trois secteurs se partagent actuellement le champ de l'univers radiophonique : celui des radios publiques et des émetteurs généralistes, celui des privés à tendance souvent très ciblée et le «troisième secteur » 
des radios associatives, que les Anglo-saxons dénomment plus volontiers « radios communautaires ». Alors que la compétition privé/public marque toujours la radio en Europe, le «troisième secteur » s'avère pour sa part en pleine expansion, en particulier dans les pays en développement. Il y joue un rôle moteur, notamment sur le plan démocratique. Et constitue ainsi un des domaines où la radio s'assure un futur.

Vis-à-vis des minorités et des peuples en devenir, la fonction sociale de la radio s'affirme de la sorte chaque jour davantage.

Dans les pays développés, l'avenir de la radio passe incontestablement par une diversification des modes d'écoute du média. Car l'audience de la radio traditionnelle s'est plutôt engagée dans une tendance baissière, et ce non seulement dans les classes les plus jeunes de la population. Tout comme le transistor sauva par le passé la radio lorsque celle-ci fut menacée par l'avènement de la télévision, les nouvelles technologies donneront demain un nouveau dynamisme au média que l'usager ne sera plus obligé d'écouter « on line ». Le podcast ainsi que tous les modes d'écoute à la demande laissent entrevoir l'organisation de nouveaux univers radiophoniques, où se redécouvriront aussi les mérites intrinsèques du sonore et des univers qu'il permet de créer. Il restera toutefois à déterminer si ces nouveaux modes d'écoute relèveront encore à l'avenir de la radiophonie ou s'ils s'inscriront davantage dans une dimension « sonore » ou « auditive » détachée des spécificités de son mode de transmission.

Bien des champs s'ouvrent donc aujourd'hui pour la recherche sur la radio. L'impression selon laquelle l'ensemble les horizons d'étude de ce média ont été atteints de longue date n'est donc pas exacte et correspond davantage à une vision dépassée du support qu'à sa prise en compte dans une perspective dynamique.

On ne peut évidemment contester qu'une mise en cadre théorique du média fut entreprise dès la genèse de la radio. Encore aujourd'hui, bon nombre de travaux scientifiques sur ce média ne peuvent éviter de circonscrire leur contexte analytique sans faire référence à la " radiotheory » des années 1930, sans citer Brecht, Benjamin et l'École de Francfort ou ceux qui, après elle, approfondirent la mise en cause de l'unilatéralité de la mass-médiatité de la radio (Enzensberger, Baudrillard...), ou sans faire allusion à ceux qui, comme Pierre Schaeffer, se plurent au contraire à souligner les caractéristiques essentielles de ce support, se 
référant tantôt à sa spécificité relationnelle, tantôt aux donnés fondamentaux de ce qui constitue le «phénomène radiophonique » : la vision sonore et la capacité de laisser entendre ${ }^{1}$.

De la même manière, il est incontestable que, de très longue date, « le langage radiophonique » a fait l'objet d'études approfondies, bon nombre de chercheurs semblant se contenter à l'heure actuelle des bornages théoriques qui en furent dressés des années 1930 aux années 1960. Quant aux études de réception, elles paraissent parfois également confiner leurs références aux premières approches, elles aussi entreprises dans les années 1940 afin de mesurer l'impact de ce «nouveau » média.

Les actuelles études et recherches sur la radio ne peuvent faire table rase ou abstraction des apports théoriques posés par le passé. Mais, tout comme il y a lieu d'étudier la radio en regard de ses domaines de développement actuel, il peut aussi y avoir lieu de remettre à l'épreuve les cadres référentiels théoriques posés par l'histoire du média.

Les contributions de ce numéro de Recherches en Communication entendront aborder ces divers aspects, tels qu'ils furent traités lors du colloque qui s'est tenu à l'UCL. Les textes ici rassemblés reflètent quelques-unes parmi la cinquantaine de communications présentées lors de ces journées ${ }^{2}$. Afin de laisser davantage d'autonomie aux auteurs, les textes ont été acceptés en français et en anglais.

C'est en 1913, c'est-à-dire bien avant qu'elles ne se répandent de par le monde, que les premières émissions régulières de radio avaient vu le jour en Belgique. Elles avaient lieu, chaque samedi, depuis le kiosque à musique du parc royal du château de Laeken. Faut-il y voir un signe au fait que, près d'un siècle plus tard, ce soit aussi, modestement, en Belgique qu'ait été relancée la recherche sur la radio, au terme des travaux du Consortium IREN?

1 Schaeffer Pierre, Propos sur la coquille, Paris, Phonurgia Nova, 1980, p. 43.

2 D'autres communications présentées lors du colloque «Radio et Recherches : quelles voies/voix pour le futur de la radio ? » ont été publiées dans le $n^{\circ} 40$ « Le retour de la radio » de la revue Médiatiques, Louvain-la-Neuve, Observatoire du Récit médiatique, printemps 2007. 